UDC: 519.688

\title{
A framework for medical image segmentation based on measuring diversity of pixel's intensity utilizing interval approach
}

\author{
A. Elaraby \\ Department of Computer Science, Faculty of Computers and Information, South Valley University, \\ H77 University st., Qena, 83523, Egypt \\ E-mail: ahmed.elaraby@svu.edu.eg
}

Received 27.04.2021, after completion - 15.06.2021. Accepted for publication 12.07.2021.

Segmentation of medical image is one of the most challenging tasks in analysis of medical image. It classifies the organs pixels or lesions from medical images background like MRI or CT scans, that is to provide critical information about the human organ's volumes and shapes. In scientific imaging field, medical imaging is considered one of the most important topics due to the rapid and continuing progress in computerized medical image visualization, advances in analysis approaches and computer-aided diagnosis. Digital image processing becomes more important in healthcare field due to the growing use of direct digital imaging systems for medical diagnostics. Due to medical imaging techniques, approaches of image processing are now applicable in medicine. Generally, various transformations will be needed to extract image data. Also, a digital image can be considered an approximation of a real situation includes some uncertainty derived from the constraints on the process of vision. Since information on the level of uncertainty will influence an expert's attitude. To address this challenge, we propose novel framework involving interval concept that consider a good tool for dealing with the uncertainty, In the proposed approach, the medical images are transformed into interval valued representation approach and entropies are defined for an image object and background. Then we determine a threshold for lower-bound image and for upper-bound image, and then calculate the mean value for the final output results. To demonstrate the effectiveness of the proposed framework, we evaluate it by using synthetic image and its ground truth. Experimental results showed how performance of the segmentation-based entropy threshold can be enhanced using proposed approach to overcome ambiguity.

Keywords: segmentation, interval arithmetic, entropy, thresholding, medical imaging

Citation: Computer Research and Modeling, 2021, vol. 13, no. 5, pp. 1059-1066. 


\section{Introduction}

Segmentation plays an important role in automated diagnostic systems in various software for medical images analysis. Medical visualization techniques development, such as magnetic resonance imaging (MRI), microscopy, ultrasound radiography, computed tomography (CT), attract investors and researchers to develop new algorithms for processing medical data. Segmentation is considered an important step in medical data visualization because it extracts the area of interest through a semiautomatic or automated process. It divides the image into regions based on a specific description, such as segmentation of body organs and tissues for border detection, tumor detection, and mass detection. Segmentation splits medical image into coherent regions, so clustering approaches can be applied by extracting global image characteristics to separate ROI data from the background. Due to the irregular and fuzzy boundaries in most medical images, the theories of fuzzy sets and neutrosophic sets become important in the process of segmentation for handling uncertainty. Therefore, the fuzzy c-means (TSM) [Kang et al., 2009] and «neutrosophic c-means clustering» (SMSS) can be applied to improve various segmentation methods. At the same time, the preliminary determination of the clusters number and their centroids is important for clustering [Ashur et al., 2018; Pei et al., 2017]. You can also use the gradient and intensity information to segment an image. There are different approaches to segmentation based on boundaries, like deformable model, and approaches based on regional methods, like region merging, active contour, and region growth [Alazawi et al., 2017; Baldevbhai et al., 2012; Sharma et al., 2010]. It can be said that most medical images have insufficient quality, noise, intensity heterogeneity, and weak boundaries, which requires complex processing [Li et al., 2011; Ma et al., 2010; Pham et al., 2000].

Developing efficient and intelligent and approaches for segmentation of medical image has become a hotspot, leading to rapidly evolving and hybrid approaches based on both boundary and region information for image segmentation are developing. Such approaches involve graph-based techniques, such as graph-cut segmentation, which is repeated to separate image background and object using explicit segmentation constraints by selecting starting points representing some pixels belonging to the background and other pixels from the object. Graphic cutting (GC) techniques are effective in segmenting medical images due to their global energy advantages. However, GC methods involve interactive object/background seed selection, which is time-consuming. To address this shortcoming, a fully automated GC procedure was developed based on mapping image data to a high dimension utilizing «Kernel Graph Reduction» (KGC) [Salah et al., 2011]. Segmentation of the skin lesion is also challenging area. Dermoscopic images have various types of lesion with several artifacts, Such artifacts increase the role of the «neutrosophic set» (NS) to handle the uncertainty during the process of segmentation which has a vital role to develop computer-aided diagnosis systems for automated skin lesion (CADs) [Celebi et al., 2009; Korotkov et al., 2012].

Most image processing algorithms supposes that a pixel represents an exact location. In practice, the data can be contaminated in various ways. In digital imaging, we can never have a full certainty about the pixel tones as many factors are to be considered like the limited number of tones, noise, or broken cells in the sensor. The uncertainty regarding the measured pixel values caused by conditions under which images are captured at which are not always ideal or stable, also in some situations is related to an image object spatial position or can be caused by technical limitations, in particular by the fact that in practice we always deal with a limited and finite number of pixels and pixel values, which leads to numerical and spatial approximations. Moreover, to be adapted for processing, an image required to be digitized [Rafael, Woods, 2001]. Usually as in nature, an image is a continuous and to convert it into a digital image it is needs a stage of approaches and consequently generator of approach errors. However, the digital image will never be an exact representation of nature even with well the resolution. Recently the concepts of fuzzy sets [Zadeh, 1965] are interlaced with intervals arithmetic [Moore, 1966, 1979]. The digital image quantification and information of how 
bad or good it was the conversion in this processing considers a fuzzy problem. Utilizing interval arithmetic for pixel representation in a continuous way it is right, and it led to improvement. We will be the sure of we be in continuous space that not always it is viable computationally to get it, it is contained.

Only few papers have dealt with the use of interval arithmetic in image processing. So, ability to propagate the uncertainty information during image processing can be important indifferent medical images tasks. Authors, presented in [Elaraby et al., 2018] an image verification framework utilizing the basic definitions to the interval digital processing; in [Elaraby et al., 2019] an edge detection of aerial images based on interval valued image approach is presented. In [Lopez-Molina et al., 2015], introduced a new approach of edge detection on interval-valued images and applied that to improve the performance of Sobel algorithm; in [Nachtegael et al., 2010] discussed the foundations of intervalvalued image model for grayscale images and the morphological theory; in [Galar et al., 2011] presented an image representing using of interval-valued fuzzy sets and its application for the task of stereo matching; in [Minamoto et al., 2010] presented a new method for digital image watermarking using interval wavelet decomposition; in [Hijazi et al., 2007] discussed the advantages of interval arithmetic in computing and its application in mathematics, computer science, and engineering; in [Takimoto et al., 2010] presented a modification for Hough transform to detecting function patterns using interval arithmetic; in [Jaulin et al., 2009] authors proposed a new method for recognition of geometrical shapes using interval.

In this paper, we introduce a framework utilizing an image representation using interval concepts, designed for better dealing with ambiguity with including entropy thresholding for reliable medical images segmentation.

\section{Proposed methodology}

Interval-Valued representation (IV) of images provides a realistic interpretation since the measurement error is important factor we should not ignore. Once we get IV-images, we can apply the threshold techniques individually on the lower or upper bounds version of an image [Elaraby et al., 2018]. In this subsection, we utilize an interval-valued representation of an image brightness that straight driven from the measurement errors analysis. That is (assuming the spatial error) we assign to each image position an interval including all the brightness values modified by \pm 1 tone «because of the tonal error».

We generate the verification interval-valued images $\mathrm{IV}_{(\mathrm{L})}, \mathrm{IV}_{(\mathrm{U})}$ and $\mathrm{IV}_{(\mathrm{M})}$ from an image $\mathrm{I}$ as:

$$
\begin{aligned}
\mathrm{IV}_{(\mathrm{L})} & =\left[\max \left(0, \mathrm{I}_{(x, y)}-1\right)\right], \\
\mathrm{IV}_{(\mathrm{U})} & =\left[\min \left(255, \mathrm{I}_{(x, y)}+1\right)\right], \\
\mathrm{IV}_{(\mathrm{M})} & =\left[\frac{\mathrm{IV}_{(\mathrm{L})}+\mathrm{IV}_{(\mathrm{U})}}{2}\right] .
\end{aligned}
$$

In image processing, entropy concept becomes increasingly important which an image can be considered as an information source [Gray, 2013; Hill, 1973; Jost, 2006; Albuquerquea et al., 2004; Naidu et al., 2018; Elaraby et al., 2017; Ishak, 2017; Bhandari et al., 2015]. In this subsection we utilized generalized «Hill-entropy».

Shannon entropy [Gray, 2013] defined as:

$$
H(p)=-\sum_{i=1}^{k} p_{i} \ln \left(p_{i}\right) .
$$


Extensive property (additive) of «Shannon» entropy is defined:

$$
H(A+B)=H(A)+H(B) .
$$

The generalized «Hill-entropy» [Hill, 1973; Jost, 2006; Evangelista et al., 2009] is defined:

$$
N_{\alpha}=\left(\sum_{i=1}^{W} p_{i}^{\alpha}\right)^{1 /(1-\alpha)} \quad \text { for } \alpha \geqslant 0 \text { and } \alpha \neq 1 .
$$

«Hill-entropy» is non-extensive with additive entropic rule:

$$
N_{\alpha}(A+B)=N_{\alpha}(A)+N_{\alpha}(B)+(1-\alpha) \cdot N_{\alpha}(A) \cdot B_{\alpha}(B) .
$$

«Hill-entropy» $N_{\alpha}(t)$ is parametrically dependent upon the threshold value $(t)$. We try to maximize the information measure between object and background, so optimum threshold value:

$$
T=\operatorname{Argmax}\left[N_{\alpha}^{A}(t)+N_{\alpha}^{B}(t)+(1-\alpha) \cdot N_{\alpha}^{A}(t) \cdot N_{\alpha}^{B}(t)\right] .
$$

In the following, figure 1 shows the schematic overview of our approach where the different steps can be observed. Blood cell image (a) is divided into (lower bound image (b) and upper bound image (c)) following threshold values $t_{1}$ and $t_{2}$ are calculated from lower bound image (b) and upper bound image (c), respectively, by «Hill-entropy». The segment results are in (d) and (e) for every case. The final segment result (f) obtained by calculated the mean value of (d) and (e).

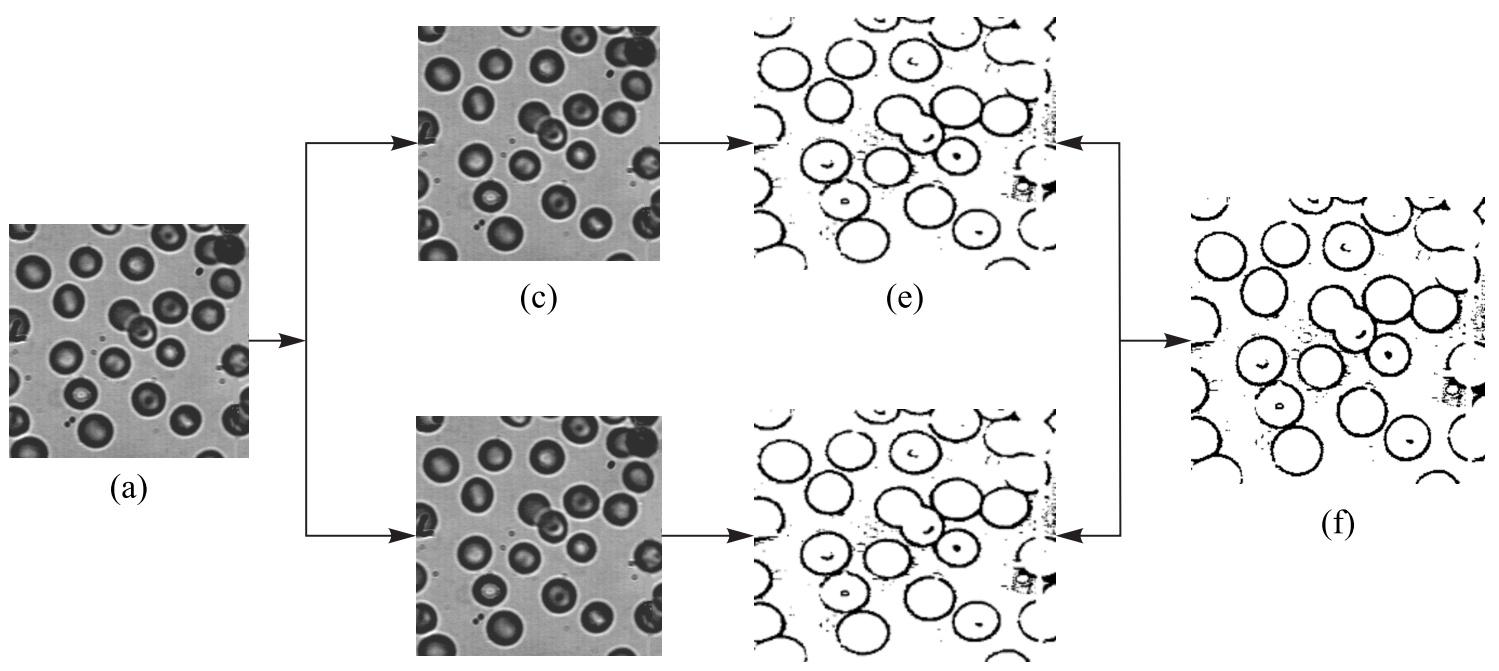

(b)

(d)

Fig. 1. Schematic overview of proposed approach: (a) blood cell image; (b) lower-bound image; (c) upper-bound image; (d) segment results of Lower-bound image; (e) segment results of upper-bound image; (f) final segment result

\section{Experimental results}

In this section, we discuss the experimental results and performance analysis obtained using proposed approach as applied to various images. This section is organized into two subsections; first, we evaluate the performance results of the proposed approach in comparison with the entropy-based segmentation by using synthetic image and its ground truth. The experiments analysis of medical images is presented in second subsection. 


\subsection{Performance evaluation using synthetic images}

We use popular objective measure which is «Dice» similarity coefficient index to measure the performance of the segmentation [Dice, 1945]. In the following figure 2, noises effects are added to synthetic images to more analysis of the performance of proposed approach. In Figure 2 shows synthetic image (a), ground truth (b), noisy synthetic image (c), Segmentation results by thresholdbased «Hill-entropy»(d), segmentation results of proposed technique (e), segmentation results of threshold-based «Hill-entropy» for noisy synthetic image (f), and segmentation results of proposed technique for noisy synthetic image (h). «Dice» values provided for the proposed approach and reference method based on synthetic images segmentation and noisy case. From the segmented results, Its observed that the proposed approach result is better than the reference method where «Dice» equals to $(0,7398)$ and «Dice» for the reference method is $(0,7320)$ for synthetic image and for noisy synthetic image, «Dice» of proposed approach is $(0,4469)$ and «Dice» of the reference method is $(0,4439)$. We can conclude that the proposed approach results are better than reference method.

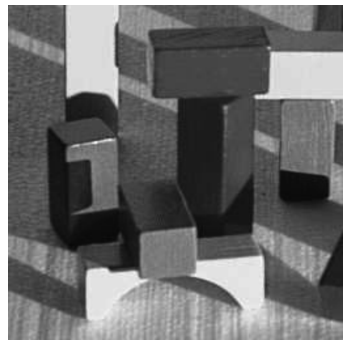

(a)

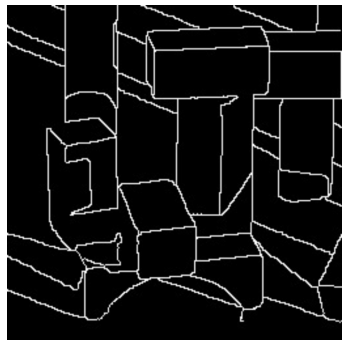

(b)

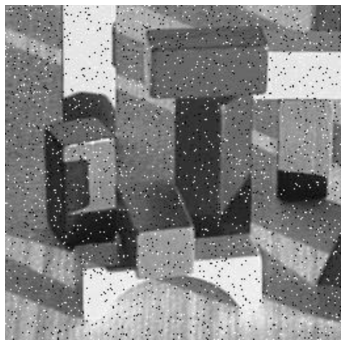

(c)

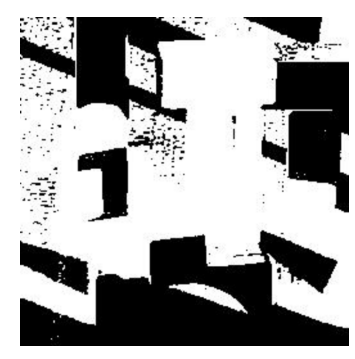

(d)

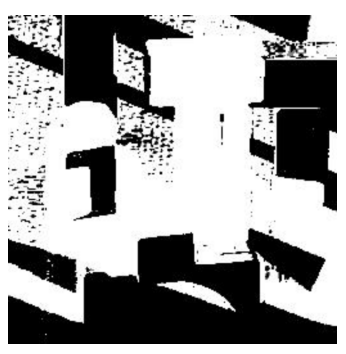

(e)

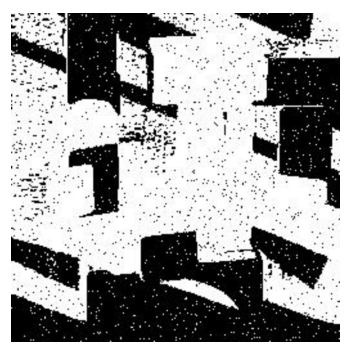

(f)

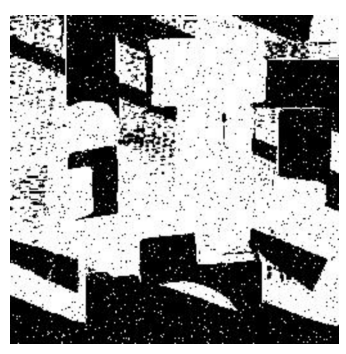

(g)

Fig. 2. Experimental results on synthetic image: (a) synthetic image; (b) ground truth; (c) noisy synthetic image; (d) segmentation results by threshold-based «Hill-entropy»; (e) segmentation results of proposed technique; (f) segmentation results of threshold-based «Hill-entropy» for noisy synthetic image; (g) segmentation results of proposed technique for noisy synthetic image

\subsection{Experimental on medical images}

The image set includes different medical images. For subjective comparison of the proposed algorithm with reference method, we applied the algorithms to the medical images set (Fig. 3). Examples of medical images (image a1, b1, c1, d1) and their histograms (image a2, b2, c2, d2), segmentation results based «Hill-entropy» approach (a3, b3, c3, d3) and proposed approach (a4, b4, $\mathrm{c} 4, \mathrm{~d} 4)$. The results indicated that the proposed approach performed better than reference method on the different medical image sets. 


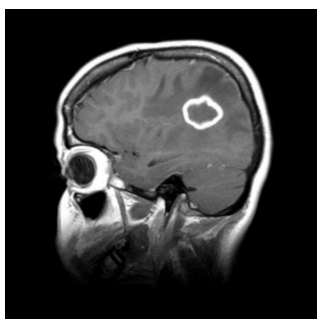

(a1)

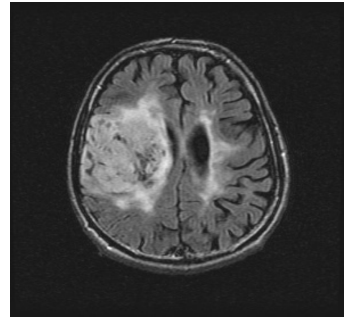

(b1)

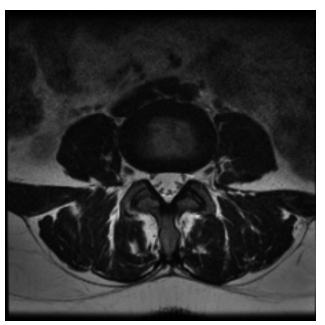

(c1)

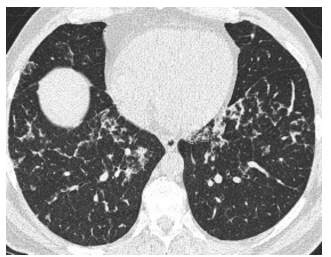

(d1)

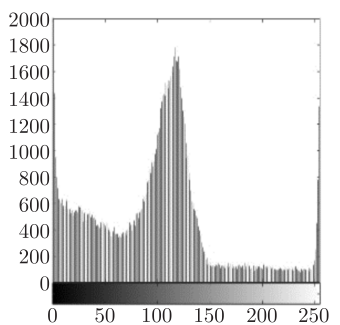

(a2)

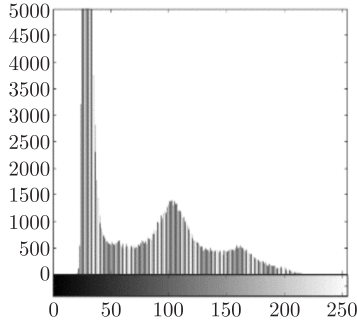

(b2)

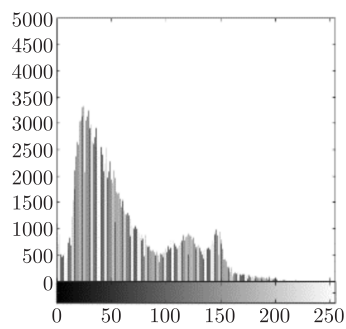

(c2)

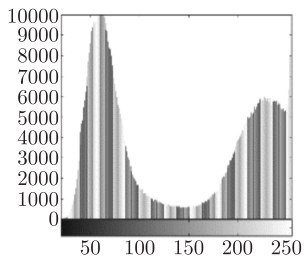

$(\mathrm{d} 2)$

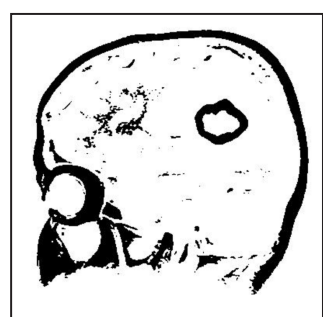

(a3)

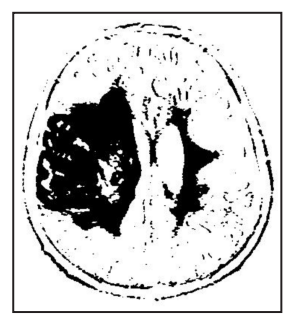

(b3)

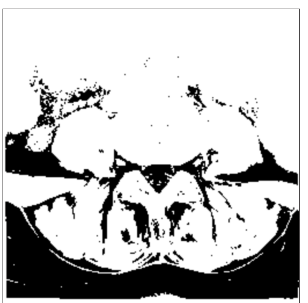

(c3)

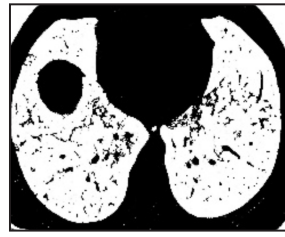

(d3)

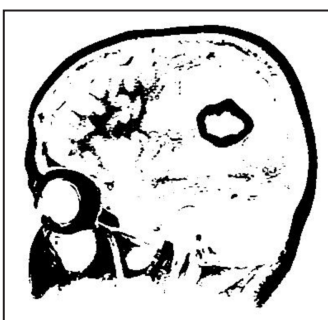

(a4)

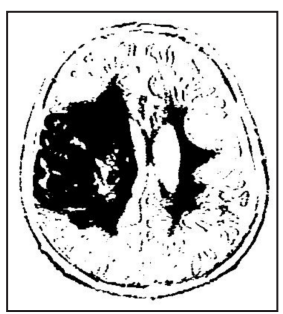

(b4)

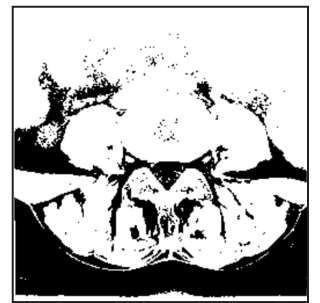

(c4)

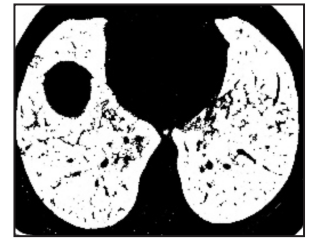

(d4)

Fig. 3. Experimental on medical images: samples of medical images (a1, b1, c1, d1) and their histograms (a2, b2, c2, d2); segmentation results based «Hill-entropy» approach (a3, b3, c3, d3) and proposed approach (a4, b4, $\mathrm{c} 4, \mathrm{~d} 4)$

\section{Conclusion}

In medical image analysis, segmentation is one of the most challenging tasks. Digital image processing becomes more important in healthcare field by the growing use of digital imaging systems for medical diagnostics. Generally, most image processing algorithms supposes that a pixel represents an exact location. In practice, the data can be contaminated in various ways. Since information on the level of uncertainty will influence an expert's attitude. we a novel framework for segmentation of medical imaged involving interval approach that consider a good tool for dealing with the uncertainty and generalized «Hill-entropy» to select threshold values required of interval valued images. Experimental results showed how the performance of the segmentation-based entropy threshold can be enhanced using proposed approach to overcome ambiguity. 


\section{References}

Al-azawi R.J., Abdulhameed A.A., Ahmed H.M. A robustness segmentation approach for skin cancer image detection based on an adaptive automatic thresholding technique // American Journal of Intelligent Systems. - 2017. - Vol. 7 (4). - P. 107-112.

Albuquerquea M.P., Esquef I.A., Gesualdi Mello A.R. Image thresholding using Tsallis entropy // Pattern Recognition Letters. - 2004. - Vol. 25, no. 9. - P. 1059-1065.

Ishak A. B. A two-dimensional multilevel thresholding method for image segmentation // Applied Soft Computing. - 2017. - Vol. 52. - P. 306-322.

Ashour A.S., Guo Y., Kucukkulahli E., Erdogmus P., Polat K. A hybrid dermoscopy images segmentation approach based on neutrosophic clustering and histogram estimation // Applied Soft Computing. - 2018. - Vol. 69. - P. 426-434.

Baldevbhai P.J., Anand R.S. Review of graph, medical and color image base segmentation techniques // IOSR Journal of Electrical and Electronics Engineering. - 2012. - Vol. 1 (1). P. 1-19.

Bhandari A., Kumar A., Singh G. Tsallis entropy based multilevel thresholding for colored satellite image segmentation using evolutionary algorithms // Expert Systems with Applications. - 2015. Vol. 42, no. 22. - P. 8707-8730.

Celebi M. E., Iyatomi H., Schaefer G., Stoecker W. V. Lesion border detection in dermoscopy images // Computerized Medical Imaging and Graphics. - 2009. - Vol. 33 (2). - P. 148-153.

Dice L.R. Measures of the amount of ecologic association between species // Ecology. - 1945. Vol. 26, no. 3. - P. 297-302.

Elaraby A., Moratal D. A Generalized Entropy-Based Two-Phase Threshold Algorithm for Noisy Medical Image Edge Detection // Scientia Iranica. - 2017. - Vol. 24, no. 6. - P. 3247-3256.

Elaraby A., Nechaevskiy A. An Interval-Valued Image Based Approach to Detect Edges in Aerial images // Proceedings of the XXVII International Symposium on Nuclear Electronics and Computing, Budva, Becici, Montenegro, 30 September - 4 October, 2019.

Elaraby A., Nechaevskiy A. An Image Verification Framework Development // Proceedings of the VIII International Conference Distributed Computing and Grid-technologies in Science and Education, Dubna, Russia, September 10-14, 2018.

Evangelista H. B. A., Thomaz S.M., Mendes R. S., Evangelista L. R. Generalized entropy inDICEs to measure $\alpha$ - and $\beta$-diversities of macrophytes // Brazilian Journal of Physics. - 2009. - Vol. 39, no. 2A. - P. 396-401.

Galar M., Barrenechea E., Fernandez J., Bustince H., Beliakov G. Representing Images By Means Of Interval-Valued Fuzzy Sets. Application to Stereo Matching // Proceedings of the IEEE Symposium on Advances in Type-2 Fuzzy Logic Systems, Paris, France, April 11-15, 2011.

Gray R. M. Entropy and Information Theory. - Springer, 2013.

Hijazi Y., Hagen H., Hansen C., Joy K.I. Why interval arithmetic is so useful // Proceedings of the Conference: Visualization of Large and Unstructured Data Sets, Kaiserslautern, Germany, September 9-11, 2007.

Hill M. O. Diversity and Evenness: A Unifying Notation and Its Consequences // Ecology. - 1973. Vol. 54. - P. 427-32.

Jaulin L., Bazeille S. Image shape extraction using interval methods // IFAC Proceedings. - 2009. Vol. 42, no. 10. - P. 378-383.

Jost L. Entropy and diversity // OIKOS. - 2006. - Vol. 113, no. 2. - P. 363-375.

Kang J., Min L., Luan Q., Li X., Liu J. Novel modified fuzzy c-means algorithm with applications // Digital Signal Processing. - 2009. - Vol. 19 (2). - P. 309-319. 
Korotkov K., Garcia R. Computerized analysis of pigmented skin lesions: a review // Artificial Intelligence in Medicine. - 2012. - Vol. 56 (2). - P. 69-90.

Li C., Huang R., Ding Z., Gatenby J., Metaxas D. N., Gore J.C. A level set method for image segmentation in the presence of intensity inhomogeneities with application to MRI // IEEE Transactions on Image Processing. - 2007. - Vol. 20 (7).

Lopez-Molina C., De Baets B., Barrenechea E., Bustince H. Edge Detection on Interval Valued Images // Advances in Intelligent and Soft Computing. - 2012. - Vol. 107. - P. 325-337.

Ma Z., Tavares J.M.R., Jorge R.N., Mascarenhas T. A review of algorithms for medical image segmentation and their applications to the female pelvic cavity // Computer Methods in Biomechanics and Biomedical Engineering. - 2010. - Vol. 13 (2). - P. 235-246.

Minamoto T., Aoki K. A blind digital image watermarking method using interval wavelet decomposition // International Journal of Signal Processing, Image Processing and Pattern Recognition. - 2010. - Vol. 3, no. 2. - P. 59-72.

Moore R. E. Interval Analysis. - New Jersey: Prentice Hall, 1966.

Moore R. E. Methods and Applications of Interval Analysis. - SIAM Studies in Applied Mathematics, 1979.

Nachtegael M., Sussner P., Melange T., Kerre E. E. Foundations of the Interval-Valued Image Model to Model and Process Uncertainty in Image Capture // Proceedings of 17th International Conference on Systems, Signals, and Image Processing, Rio de Janeiro, Brazil, June 17-19, 2010.

Naidu M.S.R., Rajesh K.P., Chiranjeevi K. Shannon and Fuzzy entropy based evolutionary image thresholding for image segmentation // Alexandria Engineering Journal. - 2018. - Vol. 57, no. 3. - P. 1643-1655.

Pei J., Zhao L., Dong X., Dong X. Effective algorithm for determining the number of clusters and its application in image segmentation // Cluster Computing. - 2017. - Vol. 20 (4). - P. 2845-2854.

Pham D. L., Xu C., Prince J.L. Current methods in medical image segmentation // Annual Review of Biomedical Engineering. - 2000. - Vol. 2 (1). - P. 315-337.

Rafael C., Woods R. C. Digital Image Processing. - Addison-Wesley Publishing Company, 2001.

Salah M. B., Mitiche A., Ayed I. B. Multiregion image segmentation by parametric kernel graph cuts // IEEE Transactions on Image Processing. - 2011. - Vol. 20 (2). - P. 545-557.

Sharma N., Aggarwal L. M. Automated medical image segmentation techniques // Journal of Medical Physics / Association of Medical Physicists of India. - 2010. - Vol. 35 (1). - P. 3.

Takimoto R. Y., Chalella das Neves A., Mafalda R., Sato A.K., Tavares R.S., Stevo N.A. Detecting Function Patterns with Interval Hough Transform // Proceedings of the IEEE International Conference on Industry Applications, Sao Paulo, Brazil, November 8-10, 2010.

Zadeh L. Fuzzy Sets // Information Control. - 1965. - Vol. 8. - P. 338-335. 